\title{
Intraoperative Fluorescence Is Useful but Not Always Sufficient in Contrast-Enhancing Malignant Gliomas
}

\author{
Aliasgar V. Moiyadi ${ }^{1}$ \\ ${ }^{1}$ Division of Neurosurgery, Department of Surgical Oncology, \\ Tata Memorial Centre, Mumbai, Maharashtra, India
}

\begin{abstract}
Address for correspondence Aliasgar V Moiyadi, MCh, Department of Neurosurgery, Tata Memorial Centre, Mumbai 400012, Maharashtra, India (e-mail: aliasgar.moiyadi@gmail.com).
\end{abstract}

\begin{abstract}
Keywords

- malignant gliomas

- fluorescence

- intraoperative ultrasound

Fluorescence-guided resections have become standard of care for malignant gliomas. Strong fluorescence has been shown to correlate with solid enhancing tumor. However, with experience it has also been shown that visualized fluorescence may not identify the entire extent of the tumor. Knowing that malignant gliomas extend beyond the contrast-enhancing tumor seen on magnetic resonance imaging (MRI), reliance only on the fluorescence intraoperatively may not be enough. Intraoperative ultrasound is a readily available tool for real-time assessment of resection status, irrespective of the tumor type. We describe one such case in which after resecting all the visible fluorescing tumor component, we identified a significant component of nonfluorescing tumor, using intraoperative ultrasound that was further resected completely. This illustrates the need for multimodal intraoperative guidance for achieving optimal tumor resection in malignant gliomas.
\end{abstract}

\section{Introduction}

Fluorescence-guided resections have become popular for contrast-enhancing malignant gliomas. This is based on the fact that contrast enhancement correlates with fluorescence and is generally proposed as a surrogate marker for selection of patients for fluorescence-guided resections (FGRs). However, relying only on the fluorescence may sometimes be insufficient. We would like to illustrate this by a case we recently encountered.

\section{Case Description}

A 56-year-old man presented with history of uncontrolled seizures of recent onset. He had no other focal neurologic deficits. Magnetic resonance imaging (MRI) of the brain revealed a focal peripherally enhancing right frontal lesion. It showed central necrosis with T2 hyperintense signal extending beyond the enhancement ( - Fig. 1). The imaging findings were consistent with a malignant glioma. Surgery was performed with pretreatment with aminolevulinic acid (Gliolan, Medac GmbH, Wedel, Germany). Navigated craniotomy was performed using a navigable three-dimensional ultrasound (3D US) system, SONOWAND (SonoWand AS, Trondheim, Norway). After durotomy, under the microscope's BLUE
400 filter (OPMI Pentero, Carl Zeiss GmbH, Oberkochen, Germany), the tumor showed bright red fluorescence when the cortex was exposed. It appeared nodular and relatively well circumscribed and was easily removed with no residual

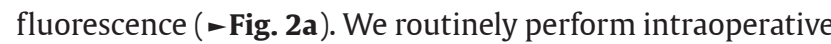
3D US for resection control in all cases. To our surprise, the postresection US revealed a sizeable residual hyperechoic tumor component ( $\mathbf{- F i g . ~ 2 b )}$ that showed no fluorescence. This was subsequently resected completely. The patient had an uneventful recovery. Postoperative MRI showed no residual tumor. The histopathology revealed typical glioblastoma features in the fluorescing component, whereas the nonfluorescing region showed features of anaplastic astrocytoma.

FGR using aminolevulinic acid (ALA) has been demonstrated to be superior to white (microscopic) light in resecting contrast enhancing malignant gliomas. Using ALA FGR, gross total resection (GTR) rates can be almost doubled, with a beneficial effect on progression-free and overall survival (though the latter has not yet been directly demonstrated)., ${ }^{1,2}$ The fluorescence has been shown to parallel tumor contrast enhancement as depicted on MRI but can be a more sensitive technique of delineating tumor extent. ${ }^{3}$ This has been shown indirectly by Schucht et al (by calculating the volume of nonenhancing tumor beyond contrast-enhancing boundaries that can be resected) ${ }^{4}$ and more received

August 7, 2017

accepted

September 4, 2017

published online

April 10, 2018
DOI https://doi.org/

$10.1055 / \mathrm{s}-0038-1639386$

ISSN 2277-954X.
(C2018 Neurological Surgeons'

Society of India
License terms

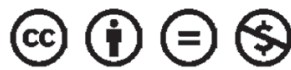




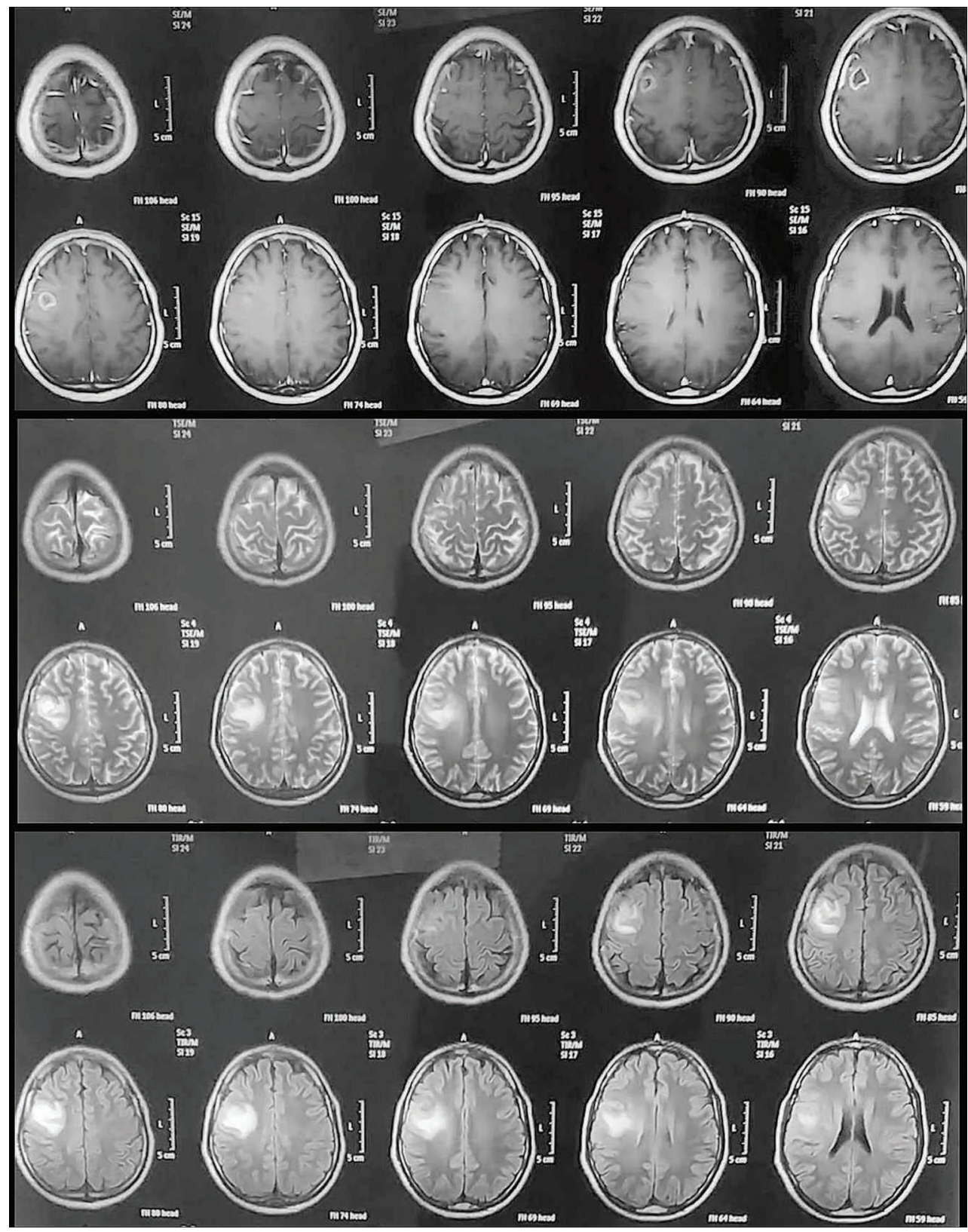

Fig. 1 Preoperative MRI of the patient showing postT1 contrast (top two rows); T2 (middle two rows); and FLAIR (bottom two rows). FLAIR, fluid-attenuated inversion recovery.

directly by Coburger et al (using a meticulous coregistration of ALA and intraoperative MRI [iMRI]). ${ }^{5}$ However, there are still limitations to this technique of fluorescence. Visualized fluorescence is a subjective interpretation, with the surgeon usually describing it as "strong” or "bright" and "weak" or "vague." Generally strong/bright fluorescence has been associated with high positive predictive value in detecting high-grade solid tumor areas. ${ }^{6}$ However, at the tumor margins the fluorescence typically seen is faint/weak/vague. This is not surprising as the border zone usually shows infiltrating, lower-grade tumor areas (even in a glioblastoma). This faintly visible fluorescence may be variably interpreted by different surgeons (making it relatively less reliable). Further, the predictive value of this faint fluorescence has been consistently shown to be less than desirably accurate. More objective and sensitive methods to record and interpret this fluorescence (spectrometry and confocal microscopy) may be useful, but they remain to be clinically validated. ${ }^{7}$ Occasionally, fluorescing tumor may be hidden behind nonfluorescing brain tissue. Therefore, absence of visualized fluorescence does not necessarily exclude tumor (the "false negatives"). Hence, some other modality/strategy may be necessary to aid tumor resection. This is illustrated by our case exemplarily. There was a significant nonfluorescing (and nonenhancing) component that would have been overlooked if not for the US. Tsugu et al have shown that in nonenhancing tumors, iMRI is better than ALA. ${ }^{8}$ Intraoperative US has long been used as an aid to localize intracranial tumors. With improved technology, navigated 3D US has been shown to be as good as iMRI in delineation of gliomas, ${ }^{9}$ especially for the nonenhancing component. Glioblastomas, though usually contrast enhancing, can have a significant nonenhancing component. The preoperative MRI T2/FLAIR is useful to identify this component. However, it may still be 

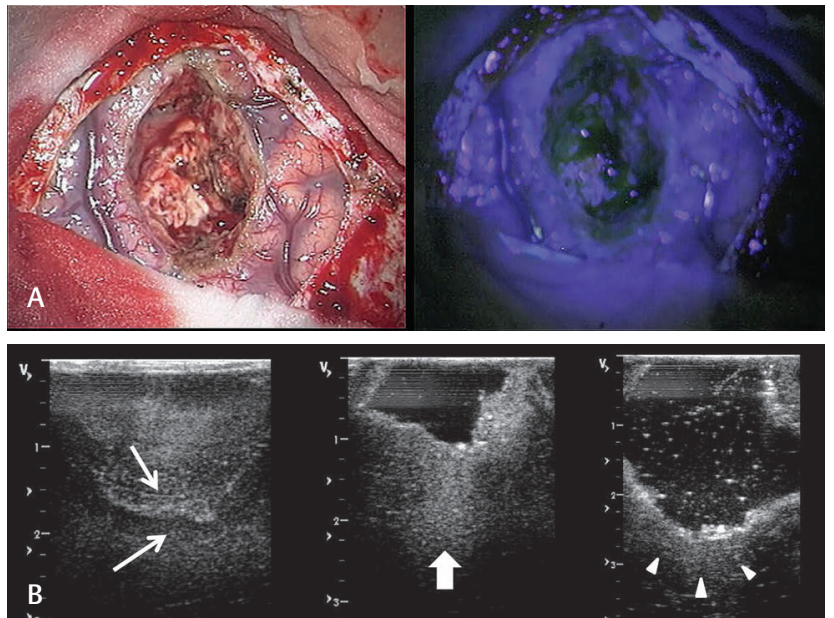

Fig. 2 (A) Intraoperative microscopic images of the operative cavity under white light (left panel) and corresponding appearance under blue filter (right panel) showing no residual fluorescence. (B) Intraoperative ultrasound findings showing pre-resection image (left) with tumor-brain interface (between the two arrows); image after resecting the fluorescing component with residual hyperechoic tumor at the base of the resection cavity (thick arrow); and final postresection image showing thin hyperechoic cavity wall (arrowheads).

difficult to differentiate from edema. As in our case, even seemingly enhancing glioblastoma multiformes (GBMs) may have a large nonenhancing part that may be overlooked unless careful attention is paid to the MRI. Only relying on the ALA fluorescence would have led to an incomplete resection in our case. We have earlier shown that ALA and US are complementary. ${ }^{10}$ This case reinforces the same conclusion and further underlines the need for a combined modality intraoperative imaging strategy even in enhancing brain tumors. The ALA fluorescence guides the resection of the viable potentially high-grade core, whereas the navigated US is helpful in localization and resection control especially in the nonenhancing border-zone as well for the deeper "invisible" tumor component (-Fig. 3). Recent evidence supports an oncologic benefit of resecting this nonenhancing component. Of course, it goes without saying that intraoperative functional mapping and monitoring is indispensable and should be used in and around eloquent areas. Judicious combination of these tools is the key to maximize benefit of resective surgery for enhancing gliomas. The aim of our report is to reiterate the fact that mere contrast enhancement can no longer be regarded as a reliable marker of tumor extent in malignant gliomas. Consequently, there is a need to combine ALA FGR with a complementary imaging adjunct to provide maximum benefit.

\section{Funding}

None.

\section{Conflict of Interest}

None.

\section{References}

1 Stummer W, Pichlmeier U, Meinel T, Wiestler OD, Zanella F, Reulen H-J; ALA-Glioma Study Group. Fluorescence-guided surgery with 5-aminolevulinic acid for resection of malignant glioma: a randomised controlled multicentre phase III trial. Lancet Oncol 2006;7(5):392-401

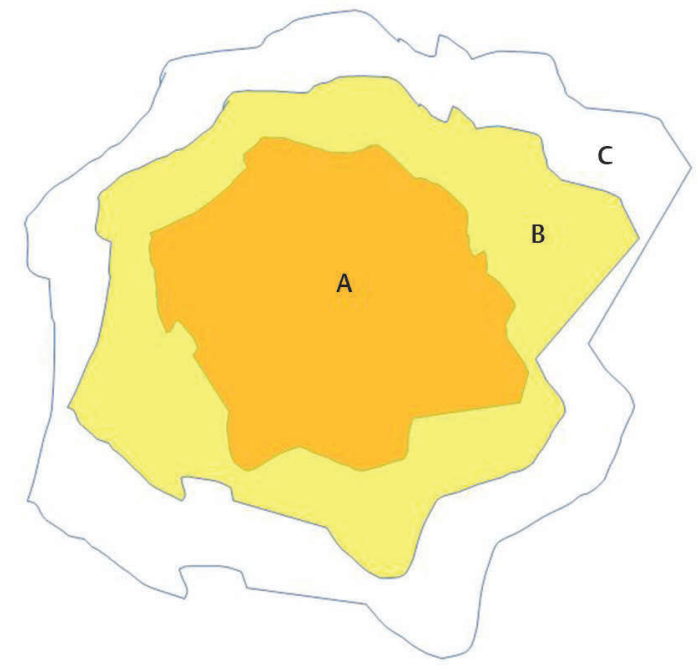

Fig. 3 Diagrammatic depiction of the various tumor components in a typical glioblastoma-A corresponds to the central enhancing tumor; $\mathrm{B}$ corresponds to nonenhancing fluorescing tumor around it; and $C$ corresponds to nonenhancing, nonfluorescing tumor. Whereas ALA would be sufficient for $A$ and $B$, ultrasound is essential for removing C. ALA, aminolevulinic acid.

2 Aldave G, Tejada S, Pay E, et al. Prognostic value of residual fluorescent tissue in glioblastoma patients after gross total resection in 5-aminolevulinic Acid-guided surgery. Neurosurgery 2013;72(6):915-920, discussion 920-921

3 Roberts DW, Valdés PA, Harris BT, et al. Coregistered fluorescence-enhanced tumor resection of malignant glioma: relationships between $\delta$-aminolevulinic acid-induced protoporphyrin IX fluorescence, magnetic resonance imaging enhancement, and neuropathological parameters. Clinical article. J Neurosurg 2011;114(3):595-603

4 Schucht P, Knittel S, Slotboom J, et al. 5-ALA complete resections go beyond MR contrast enhancement: shift corrected volumetric analysis of the extent of resection in surgery for glioblastoma. Acta Neurochir (Wien) 2014;156(2):305-312, discussion 312

5 Coburger J, Engelke J, Scheuerle A, et al. Tumor detection with 5-aminolevulinic acid fluorescence and Gd-DTPA-enhanced intraoperative MRI at the border of contrast-enhancing lesions: a prospective study based on histopathological assessment. Neurosurg Focus 2014;36(2):E3

6 Stummer W, Tonn JC, Goetz C, et al. 5-Aminolevulinic acid-derived tumor fluorescence: the diagnostic accuracy of visible fluorescence qualities as corroborated by spectrometry and histology and postoperative imaging. Neurosurgery 2014;74(3):310-319, discussion 319-320

7 Sanai N, Snyder LA, Honea NJ, et al. Intraoperative confocal microscopy in the visualization of 5-aminolevulinic acid fluorescence in low-grade gliomas. J Neurosurg 2011; 115(4):740-748

8 Tsugu A, Ishizaka H, Mizokami Y, et al. Impact of the combination of 5-aminolevulinic acid-induced fluorescence with intraoperative magnetic resonance imaging-guided surgery for glioma. World Neurosurg 2011;76(1-2):120-127

9 Unsgaard G, Selbekk T, Brostrup Müller T, et al. Ability of navigated 3D ultrasound to delineate gliomas and metastases-comparison of image interpretations with histopathology. Acta Neurochir (Wien) 2005;147(12):1259-1269, discussion 1269

10 Moiyadi A, Shetty P. Navigable intraoperative ultrasound and fluorescence-guided resections are complementary in resection control of malignant gliomas: one size does not fit all. J Neurol Surg A Cent Eur Neurosurg 2014;75(6):434-441 